\begin{tabular}{|c|c|}
\hline Title & $\begin{array}{l}\text { Dynamic pathways to mediate reactions buried in thermal fluctuations. I. Time dependent normal form theory for } \\
\text { multidimensional Langevin equation }\end{array}$ \\
\hline Author(s) & Kawai, Shinnosuke; Komatsuzaki, Tamiki \\
\hline Citation & $\begin{array}{l}\text { Journal of Chemical Physics, 131(22), } 224505 \\
\text { https://doi.org/10.1063/1.3268621 }\end{array}$ \\
\hline Issue Date & 2009-12-14 \\
\hline Doc URL & http:/hdl. handle.net/2115/42520 \\
\hline Rights & $\begin{array}{l}\text { Copyright } 2009 \text { A merican Institute of Physics. This article may be downloaded for personal use only. A ny other use } \\
\text { requires prior permission of the author and the A merican Institute of Physics. The following article appeared in J. } \\
\text { Chem. Phys. 131, 224505 (2009) and may be found at https://dx.doi.org/10.1063/.3268621 }\end{array}$ \\
\hline Type & article \\
\hline File Information & JCP131-22_224505.pdf \\
\hline
\end{tabular}

Instructions for use 


\title{
Dynamic pathways to mediate reactions buried in thermal fluctuations. I. Time-dependent normal form theory for multidimensional Langevin equation
}

\author{
Shinnosuke Kawai ${ }^{1, \text { a) }}$ and Tamiki Komatsuzaki ${ }^{1,2}$ \\ ${ }^{1}$ Molecule and Life Nonlinear Sciences Laboratory, Research Institute for Electronic Science, Hokkaido \\ University, Kita 20 Nishi 10, Kita-ku, Sapporo 001-0020, Japan \\ ${ }^{2}$ Core Research for Evolutional Science and Technology (CREST), Japan Science and Technology Agency \\ (JST), Kawaguchi, Saitama 332-0012, Japan
}

(Received 6 October 2009; accepted 6 November 2009; published online 9 December 2009)

\begin{abstract}
We present a novel theory which enables us to explore the mechanism of reaction selectivity and robust functions in complex systems persisting under thermal fluctuation. The theory constructs a nonlinear coordinate transformation so that the equation of motion for the new reaction coordinate is independent of the other nonreactive coordinates in the presence of thermal fluctuation. In this article we suppose that reacting systems subject to thermal noise are described by a multidimensional Langevin equation without a priori assumption for the form of potential. The reaction coordinate is composed not only of all the coordinates and velocities associated with the system (solute) but also of the random force exerted by the environment (solvent) with friction constants. The sign of the reaction coordinate at any instantaneous moment in the region of a saddle determines the fate of the reaction, i.e., whether the reaction will proceed through to the products or go back to the reactants. By assuming the statistical properties of the random force, one can know a priori a well-defined boundary of the reaction which separates the full position-velocity space in the saddle region into mainly reactive and mainly nonreactive regions even under thermal fluctuation. The analytical expression of the reaction coordinate provides the firm foundation on the mechanism of how and why reaction proceeds in thermal fluctuating environments. (C) 2009 American Institute of Physics. [doi:10.1063/1.3268621]
\end{abstract}

\section{INTRODUCTION}

How a system can robustly undergo a reaction from one stable state to another in the presence of thermal fluctuations has been one of the most intriguing subjects in chemistry and biology. Most reactions in biology or chemical synthesis occur in condensed phase. The most characteristic difference from the isolated system is that the system (e.g., solute) is subject to external forces exerted by the surrounding environment (e.g., solvents). Even for a fixed initial condition of all the variables in the system, the final destination is not necessarily unique but has a certain probability distribution because one cannot control the external force due to its stochastic nature. In the pioneering studies by Kramers ${ }^{1}$ (and later by Grote and $\mathrm{Hynes}^{2}$ ), condensed phase reactions are described in terms of (generalized) Langevin equations in which the system moves along a one-dimensional coordinate (in the configuration space) on the potential (of mean force) under the influence of friction (friction kernel) and the random force exerted by the environment. The system-bath Hamiltonian approach can formally bridge the descriptions of any Hamiltonian system and the generalized Langevin formulation $^{3-5}$ projected onto an arbitrarily chosen coordinate. The question of the description of dynamical systems in the presence of thermal fluctuation is one of the most intriguing subjects in nonequilibrium statistical mechanics. ${ }^{6,7}$ In ad-

${ }^{a)}$ Electronic mail: skawai@es.hokudai.ac.jp. dition, there remains the fundamental question of what reaction coordinate a system actually follows under the disturbance of thermal fluctuation.

A concept of "reaction coordinate" has long been envisioned as a one-dimensional coordinate that describes the progress of the reaction. The coordinate is considered to lie, at least approximately, along the unstable direction in the vicinity of the saddle point of rank one (associated with one negative eigenvalue of Hessian matrix while all the others are positive), going from the reactant to the product region. If there were no coupling between the reaction coordinate and all the other coordinates, the process of the reaction could be described as a one-dimensional system with that coordinate. That is, it could be predicted whether the system will go forward to the product or back to the reactant by referring solely to the value of the reaction coordinate, without referring to the rest. For molecules with many atoms, however, the couplings among multiple modes make the reaction dynamics much more complicated. It is far from trivial whether such a reaction coordinate, decoupled from all the other coordinates, actually exists in nonlinearly coupled multidimensional systems surrounded by a thermally fluctuating environment.

In isolated systems with multiple degrees of freedom, nonlinear dynamics in the region of rank-one saddle was recently studied by several authors, ${ }^{8-26}$ following several developments in experiments ${ }^{27,28}$ and theories. ${ }^{29-38}$ The firm 
mathematical foundation has been established, for the existence of the reaction coordinate decoupled from the rest, as a nonlinear function of the original coordinates and the conjugate momenta in the phase space, even though there exists nonlinear couplings among the original coordinates ${ }^{8-26}$ (one can also see several reviews ${ }^{39-46}$ and books ${ }^{47,48}$ ). The crux was the nonlinear transformation of the coordinate systems by using the normal form (NF) theory, which uses the Lie canonical perturbation theory (regarded as a classical analog of the Van Vleck perturbation theory in quantum mechanics). ${ }^{49,50}$ The occurrence of the reaction can then be judged solely from the value of the reaction coordinate, irrespective of all the nonreactive modes. However, their approach is based on Hamiltonian systems, which corresponds to "isolated molecules."

In the framework of multidimensional underdamped Langevin equation, Bartsch et al. ${ }^{51-53}$ recently discussed the potential of the introduction of a shifted coordinate system that takes into account time-dependent fluctuating forces and showed that the shifted coordinate system became apparently free from stochastic thermal fluctuation, and one could extract the geometrical structure such as the reaction coordinate decoupled from the other nonreactive coordinates in that shifted system. However, the separation of the reaction coordinate from the others crucially results from the harmonic approximation for the potential they used. The harmonic approximation, however, is only validated in close proximity of the saddle point. At higher temperature the system more significantly experiences nonlinear regions on the potential, which invalidates their treatments. There still remains an unresolved question of the existence of a clear reaction boundary or reaction coordinate, which enables us to predict the final destination of the reaction under thermal fluctuation.

In this article we present a theory to single out a new reaction coordinate decoupled from the other nonreactive coordinates in the presence of thermal fluctuations without postulating a priori either the dimension or the form of the potential (of mean force) (it can be multidimensional and nonlinear). Here we assume that reacting systems are described by the framework of multidimensional (underdamped) Langevin equation. The term "underdamped" means that the second time derivative of the position $\boldsymbol{q}$ is included in the equation of motion, which is distinguished from the "overdamped" case of $\ddot{q}=0$, where equilibration in the velocity $(\dot{q})$ space is supposed to be attained much more quickly than in the position $(\boldsymbol{q})$ space. The crux is the application of the non-Hamiltonian NF theory ${ }^{54}$ together with the time-dependent formulation given by Ref. 55. We show that the sign of the new coordinate at any instantaneous moment in the saddle region, in principle, determines the fate of the reaction: That is whether the system reaction will go forward to the products or go back to the reactants. By assuming the statistical properties of the random force, one can know a priori a well-defined boundary of the reaction which separates the full position-velocity space (spanned by the position $\boldsymbol{q}$ and the velocity $\dot{\boldsymbol{q}}$ ) in the saddle region into mainly reactive and mainly nonreactive regions even under thermal fluctuation. In addition to obtaining the reaction boundary, the introduction of the new coordinate provides detailed insights into the flows of trajectories in the position-velocity space. The analytical expression for the new reaction coordinate in terms of $(\boldsymbol{q}, \dot{\boldsymbol{q}})$ of the system and the random force $\boldsymbol{\xi}(t)$ from the solvent enables us to obtain physical insights about the pathway to mediate the reaction.

The main interest of this article is the question of the general existence of a reaction coordinate in the positionvelocity space by which one can predict the destination of a reaction in a thermally fluctuating environment. For simplicity, our theory will be presented in the framework of the multidimensional underdamped Langevin formulation (the extension to the generalized Langevin formulation is rather straightforward and will be presented elsewhere). Here we mainly focus on the description of the theoretical framework. The actual application of our theory will be presented using the Müller-Brown potential ${ }^{56}$ in a separate paper. ${ }^{60}$

\section{THEORY}

The reaction process under a fluctuating environment is described by multidimensional underdamped Langevin equation,

$$
\ddot{q}_{j}=-\frac{\partial U}{\partial q_{j}}-\sum_{i=1}^{n} \gamma_{i j} \dot{q}_{i}+\xi_{j}(t),
$$

where $\left(q_{1}, q_{2}, \ldots, q_{n}\right)$ are position coordinates of the system, with $n$ being the number of degrees of freedom of the system, $U$ the potential (of mean force), $\gamma_{i j}$ 's are friction coefficients, and $\xi_{j}(t)$ 's describe the random force from the fluctuating environment. We placed no assumption about the dimensionality of the system, the form of the friction constants [i.e., whether the matrix $\boldsymbol{\Gamma}=\left(\gamma_{i j}\right)$ has off-diagonal elements or not], or the form of potential, except that the system possesses a rank-1 saddle point that links the reactant and the product regions (see Sec. II A for more detail). Without loss of generality, the origin $(\boldsymbol{q}=\mathbf{0})$ is set to be at the saddle point. The fluctuating force $\xi_{j}(t)$ has a Gaussian distribution with zero mean, their variance being given by the fluctuationdissipation theorem,

$$
\left\langle\xi_{i}(t) \xi_{j}\left(t^{\prime}\right)\right\rangle=2 k_{\mathrm{B}} T \gamma_{i j} \delta\left(t-t^{\prime}\right),
$$

where $T$ is the temperature, $k_{\mathrm{B}}$ is the Boltzmann constant, and $\delta$ is Dirac's delta function, respectively.

The overall goal of this section is to construct a coordinate transformation $\left(q_{1}, \ldots, q_{n}, \dot{q}_{1}, \ldots, \dot{q}_{n}\right) \mapsto\left(y_{1}, \ldots, y_{2 n}\right)$, which casts the equations of motion into the following form:

$$
\begin{aligned}
& \dot{y}_{1} \approx\left\{\lambda_{1}+c_{1}(t)\right\} y_{1}, \\
& \dot{y}_{j} \approx \lambda_{j} y_{j}+c_{j}\left(y_{1}, \ldots, y_{2 n}, t\right), \quad j=2,3, \ldots, 2 n,
\end{aligned}
$$

where $\lambda_{1}$ is a positive number determined by the curvature along the unstable direction and the friction coefficient(s), and $\left|c_{1}(t)\right|$ is smaller than $\lambda_{1}$, that is, the motion along the $y_{1}$-direction is unstable. The unstable direction corresponds to the motion sliding down the barrier, whereas all the other directions are stable. The time scale $\lambda_{1}$ of the unstable motion is determined by the potential $U(\boldsymbol{q})$ and the friction constants $\gamma_{i j}$ (details are given in Sec. II A and Appendix A). If there is no friction, it is equal to the square root of the cur- 
vature of the $U(\boldsymbol{q})$ along the unstable direction. Existence of the friction decreases the instability of the system and, in other words, prevents the system from sliding down away from the saddle, resulting in smaller value of $\lambda_{1}$ (Appendix A). The term $c_{1}(t)$ originates from the nonlinearity of the potential and the random force (details in Sec. II C), whose time dependence is due to the time dependence of the random force. The physical interpretation is that the random force (the kick from the environment) disturbs the position of the system on the surface of $U(\boldsymbol{q})$, resulting in a change in the effective curvature along the reaction coordinate through the nonlinearity of the potential. In what follows, we regard the harmonic part (the quadratic terms in the Taylor expansion of the potential $U$ ) as the zeroth order approximation and treat the higher-order terms (i.e., nonlinearity) as perturbations. As long as this treatment is valid, the term $c_{1}(t)$ can usually be expected to be smaller than $\lambda_{1}$ since it comes from perturbational terms.

In Eq. (3), it is seen that the motion of $y_{1}$ is decoupled from all the other coordinates (see Sec. II D for details). If $y_{1}$ has a positive (negative) value in the vicinity of the saddle, the sign remains positive (negative) for all the time. The most striking point is that the sign of $y_{1}$ solely determines to which direction the system departs from the barrier, irrespective of the other modes $\left(y_{2}, y_{3}, \ldots\right)$, even though the latter can be coupled to each other, as we allow for any functional form for $c_{j}$ in Eq. (3). In other words, knowing the sign of $y_{1}$ at any instant in the vicinity of the saddle is sufficient to predict whether the system will reach the product or bounce back to the reactant.

The construction of the transformation follows three steps: diagonalization of the linear part (Sec. II A), shift of the origin (Sec. II B), and nonlinear transformation to incorporate the effect of nonlinear couplings (Sec. II C).

\section{A. Diagonalization of linear part}

First we perform the Taylor expansion of the force $\left(-\partial U / \partial q_{j}\right)$ and decompose it into linear and nonlinear parts,

$$
-\frac{\partial U}{\partial q_{j}}=-\sum_{i=1}^{n} k_{i j} q_{i}+\sum_{k=1}^{\infty} \varepsilon^{k} \sum_{|\boldsymbol{m}|=k+1} \alpha_{j, m} q_{1}^{m_{1}} \ldots q_{n}^{m_{n}},
$$

where $k_{i j}$ and $\alpha_{j, m}$ are the expansion coefficients of linear and nonlinear parts, respectively. In Eq. (4) we have introduced a formal parameter $\varepsilon$ because we will later incorporate the effect of higher-order terms by time-dependent NF perturbation theory. After all the calculations are carried out, we can set $\varepsilon=1$. The perturbation order $k$ is assigned according to the total power of $q_{j}$ 's. Thus the linear part is zeroth order and the $k$ th order term consists of homogeneous polynomials with

$$
|\boldsymbol{m}|=\sum_{j=1}^{\operatorname{def}} m_{j}=k+1,
$$

where $m_{j}$ is the power of $q_{j}$ in the expansion Eq. (4).

Some of the nonlinear terms in Eq. (4) include coordinates $q_{i}$ with $i \neq j$. This means that the motion along the $j$ th direction is affected by the position along other modes $q_{i}(i \neq j)$, and therefore the motion of each mode cannot be described independently from others. The effect is called "nonlinear coupling" or simply "coupling" between the $j$ th and $i$ th modes. The nonlinear terms also include terms such as $q_{j}^{2}, q_{j}^{3}, \ldots$, without $q_{i}(i \neq j)$, that is, nonlinearity along one single direction. Both types of nonlinearity cause deviation from purely harmonic motion, which will be treated in Sec. II C. Here let us begin to investigate the linear part of the equation.

The Langevin equation [Eq. (1)] can be rewritten into a first order differential equation in the position-velocity space

$$
\frac{d}{d t}\left(\begin{array}{l}
\boldsymbol{q} \\
\dot{\boldsymbol{q}}
\end{array}\right)=\left(\begin{array}{cc}
\mathbf{0} & \mathbf{1} \\
-\boldsymbol{K} & -\boldsymbol{\Gamma}
\end{array}\right)\left(\begin{array}{c}
\boldsymbol{q} \\
\dot{\boldsymbol{q}}
\end{array}\right)+\left(\begin{array}{c}
\mathbf{0} \\
\boldsymbol{\xi}(t)
\end{array}\right)+O(\varepsilon),
$$

where $\boldsymbol{K}$ and $\boldsymbol{\Gamma}$ are $n \times n$ matrices with components $k_{i j}$ and $\gamma_{i j}$, respectively. We have assumed that the system in question has a rank-one saddle point to link the reactant and the product regions. "Rank-one saddle point" here means that one of the eigenvalues for the linear part of Eq. (1) has a positive real value. That is, the matrix can be diagonalized with only one positive real eigenvalue,

$$
\begin{aligned}
& \boldsymbol{V}^{-1}\left(\begin{array}{cc}
\mathbf{0} & \mathbf{1} \\
-\boldsymbol{K} & -\boldsymbol{\Gamma}
\end{array}\right) \boldsymbol{V}=\operatorname{diag}\left(\lambda_{1}, \lambda_{2}, \ldots, \lambda_{2 n}\right), \\
& \lambda_{1}>0, \\
& \operatorname{Re}\left(\lambda_{j}\right)<0, \quad j=2, \ldots, 2 n,
\end{aligned}
$$

where $\boldsymbol{V}$ is a $2 n \times 2 n$ matrix. Hereinafter we designate the positive eigenvalue as $\lambda_{1}$. In the case of isotropic friction, we also have one negative real eigenvalue (Appendix A), which we designate as $\lambda_{2}$. As one can see here, the rank-one saddle point we defined includes both the information of the linear parts of potential (i.e., the curvature of the potential) and friction constants. If the friction is isotropic, i.e., $\gamma_{i j}=\gamma_{i i} \delta_{i j}$, the rank-1 saddle corresponds to a saddle point of the potential, but, in general, this is not necessarily so.

When we introduce normal mode coordinates $u_{j}$ in the position-velocity space by

$$
\left(\begin{array}{l}
\boldsymbol{q} \\
\dot{\boldsymbol{q}}
\end{array}\right)=\boldsymbol{V u} \text {. }
$$

The equation of motion is cast into the following form:

$$
\frac{d}{d t} u_{j}=\lambda_{j} u_{j}+\widetilde{\xi}_{j}(t)+\sum_{k=1}^{\infty} \varepsilon^{k} \sum_{|\boldsymbol{m}|=k+1} \beta_{j, m} u_{1}^{m_{1}} \ldots u_{2 n}^{m_{2 n}}
$$

where $\widetilde{\xi}_{j}(t)$ is the $j$ th component of the following vector:

$$
\widetilde{\boldsymbol{\xi}}=\boldsymbol{V}^{-1}\left(\begin{array}{c}
\mathbf{0} \\
\boldsymbol{\xi}(t)
\end{array}\right),
$$

and $\beta_{j, \boldsymbol{m}}$ 's represent the nonlinearity whose order is $k=|\boldsymbol{m}|$ -1 . In linear approximation $(\varepsilon \rightarrow 0)$, the motion of $u_{j}$ only depends on $u_{j}$ itself and the random force exerted by the fluctuating environment. This means that the normal modes $\boldsymbol{u}$ are "decoupled" from each other in the lowest order even though the external force exerted by solvent kicks each normal mode $u_{j}$ stochastically. The higher-order terms [the third 
terms in Eq. (9)] break down this separation and this effect will be treated in Sec. II C. Before taking into account the nonlinearity of the system, we treat the random force $\widetilde{\boldsymbol{\xi}}(t)$ using the prescription proposed by Bartsch et al. ${ }^{51-53}$ in Sec. II B.

\section{B. Shift of origin}

Bartsch et al. ${ }^{51-53}$ suggested a time-dependent shift of origin and introduced relative coordinates, which we call $x_{j}$ here, by

$$
\begin{aligned}
& u_{j}=u_{j}^{\ddagger}(t)+x_{j}, \\
& u_{j}^{\ddagger}(t)= \begin{cases}\int_{-\infty}^{0} \exp \left(-\lambda_{j} \tau\right) \tilde{\xi}_{j}(t+\tau) d \tau & \left(\operatorname{Re} \lambda_{j}<0\right) \\
-\int_{0}^{+\infty} \exp \left(-\lambda_{j} \tau\right) \tilde{\xi}_{j}(t+\tau) d \tau & \left(\operatorname{Re} \lambda_{j}>0\right) .\end{cases}
\end{aligned}
$$

When Eq. (11) is substituted into Eq. (9), the full equation of motion becomes

$$
\begin{aligned}
& \frac{d}{d t} x_{j}=\lambda_{j} x_{j}+f_{j}(\boldsymbol{x}, t), \\
& f_{j}(\boldsymbol{x}, t)=\sum_{k=1}^{\infty} \varepsilon^{k} f_{j}^{(k)}(\boldsymbol{x}, t), \\
& f_{j}^{(k)}(\boldsymbol{x}, t)=\sum_{|\boldsymbol{m}| \leq k+1} f_{j, \boldsymbol{m}}^{(k)}(t) x_{1}{ }^{m_{1}}, \ldots, x_{2 n}{ }^{m_{2 n}},
\end{aligned}
$$

where $f_{j, m}^{(k)}(t)$ are time-dependent coefficients which depend on $\beta_{j, m}$ in Eq. (9) and $u_{j}^{\ddagger}(t)$. Note that in the lowest order the dynamics of $x_{j}$ does not depend on $\widetilde{\xi}_{j}$ because it is canceled out by the shift, ${ }^{51-53}$ but in the higher-order terms of $O\left(\varepsilon^{k}\right)$ $(k \geq 1)$ the dynamics of $x_{j}$ depends on all $\tilde{\xi}$ through the time dependence in $f_{j}^{(k)}(\boldsymbol{x}, t)$ that originates from that of random force $\boldsymbol{\xi}(t)$. Hereinafter for the sake of brevity the dependence on $\xi_{j}(t)$ and $\gamma_{i j}$ is not explicitly written in the notations of the time-dependent coefficients appearing in the following procedures [i.e., $c_{j}(\boldsymbol{y}, t), w_{j}(\boldsymbol{y}, t)$, and $\left.g_{j}(\boldsymbol{y}, t)\right]$ but all these coefficients generally depend on both $\xi_{j}(t)$ and $\gamma_{i j}$ exerted by the surrounding environment.

It is convenient for later use to introduce $S$-symbols defined in Ref. 55,

$$
S[\mu, \psi](t)= \begin{cases}\int_{-\infty}^{0} \exp (-\mu \tau) \psi(t+\tau) d \tau & (\operatorname{Re} \mu<0) \\ -\int_{0}^{+\infty} \exp (-\mu \tau) \psi(t+\tau) d \tau & (\operatorname{Re} \mu>0)\end{cases}
$$

for any complex value $\mu$ and function $\psi(t)$. This symbol is defined so that it satisfies the following differential equation:

$$
\left(\frac{d}{d t}-\mu\right) S[\mu, \psi](t)=\psi(t) .
$$

With this symbol, we can rewrite Eq. (11) as

$$
u_{j}^{\ddagger}(t)=S\left[\lambda_{j}, \tilde{\xi}_{j}\right](t) .
$$

The following properties ${ }^{55}$ of $S$-symbol can easily be shown and will be useful in the symbolic calculation in Sec. II C: For any functions $\psi(t), \varphi(t)$, and constants $a, b, \mu$, and $\nu(\in \mathrm{C})$,

$$
\begin{aligned}
& S[\mu, a \psi+b \varphi]=a S[\mu, \psi]+b S[\mu, \varphi], \\
& S[\mu, S[\lambda, \psi]]=S[\lambda, S[\mu, \psi]]=\frac{1}{\mu-\lambda}(S[\mu, \psi]-S[\lambda, \psi]), \\
& S[\mu, 1]=-\frac{1}{\mu} .
\end{aligned}
$$

A remark on the differentiabilities of the random force $\boldsymbol{\xi}(t)$ and the $S$-symbol may be beneficial. The random force $\boldsymbol{\xi}(t)$ is a generalized function that has infinite root mean square [Eq. (2)]. Since the integration of $\boldsymbol{\xi}(t)$ becomes a random walk, it increases proportionally to the square root of time,

$$
\int_{t}^{t+\Delta t} \xi_{j}(\tau) d \tau=O\left(\Delta t^{1 / 2}\right)
$$

From the definition of $S$-functionals [Eq. (13)], it is readily shown that

$$
S\left[\mu, \xi_{j}\right](t+\Delta t)-S\left[\mu, \xi_{j}\right](t)=O\left(\Delta t^{1 / 2}\right)
$$

for any complex number $\mu$ with $\operatorname{Re} \mu \neq 0$. This means that $S\left[\mu, \xi_{j}\right]$ is a continuous function of $t$,

$$
S\left[\mu, \xi_{j}\right] \in C^{0},
$$

where $C^{0}$ is a set of continuous functions. In general, the differentiability increases by one after the application of $S$ because of Eq. (14). Namely,

$$
\psi \in C^{k} \Rightarrow S[\mu, \psi] \in C^{k+1},
$$

where $C^{k}$ is a set of $k$ times differentiable functions, and $\psi$ is any function in $C^{k}$. While the random force is not a continuous function, the shift applied in Eq. (11) is continuous in $t$.

\section{Time-dependent normal form theory}

To incorporate the effect of the nonlinear terms remaining in Eq. (12), we generalize the NF theory given by Leung and Zhang ${ }^{54}$ into time-dependent form. We start with the equation of motion for $x_{j}$ [Eq. (12)] and introduce a new set of coordinates $y_{j}$ by

$$
\begin{aligned}
& x_{j}=y_{j}+w_{j}(\boldsymbol{y}, t), \\
& w_{j}(\boldsymbol{y}, t)=\sum_{k=1}^{\infty} \varepsilon^{k} w_{j}^{(k)}(\boldsymbol{y}, t),
\end{aligned}
$$

with the functional forms of $w_{j}^{(k)}(\boldsymbol{y}, t)$ being determined later. The equation of motion for $y_{j}$, then, has the following form: 


$$
\begin{aligned}
& \frac{d}{d t} y_{j}=\lambda_{j} y_{j}+c_{j}(\boldsymbol{y}, t), \\
& c_{j}(\boldsymbol{y}, t)=\sum_{k=1}^{\infty} \varepsilon^{k} c_{j}^{(k)}(\boldsymbol{y}, t) .
\end{aligned}
$$

Here the functional forms of $c_{j}^{(k)}(\boldsymbol{y}, t)$ depend on $w_{j}^{(k)}(\boldsymbol{y}, t)$. We determine the functions $w_{j}^{(k)}(\boldsymbol{y}, t)$ so that the final equation of motion for $y_{1}$ contains no coupling term up to a certain order in $\varepsilon$, as was stated in Eq. (3). When we substitute Eqs. (21) and (22) into Eq. (12), we obtain

$$
\begin{aligned}
& \sum_{i=1}^{2 n} \lambda_{i} y_{i} \frac{\partial}{\partial y_{i}} w_{j}(\boldsymbol{y}, t)-\lambda_{j} w_{j}(\boldsymbol{y}, t)+\frac{\partial}{\partial t} w_{j}(\boldsymbol{y}, t) \\
& \quad=f_{j}(\boldsymbol{y}+\boldsymbol{w}, t)-\sum_{i=1}^{2 n} c_{i}(\boldsymbol{y}, t) \frac{\partial}{\partial y_{i}} w_{j}(\boldsymbol{y}, t)-c_{j}(\boldsymbol{y}, t) .
\end{aligned}
$$

Here we assume that the following functions can be described as polynomial expansions in $\boldsymbol{y}$ :

$$
\begin{aligned}
& w_{j}^{(k)}(\boldsymbol{y}, t)=\sum_{|\boldsymbol{m}| \leq k+1} w_{j, \boldsymbol{m}}^{(k)}(t) y_{1}^{m_{1}} \ldots y_{2 n}{ }^{m_{2 n}}, \\
& c_{j}^{(k)}(\boldsymbol{y}, t)=\sum_{|\boldsymbol{m}| \leq k+1} c_{j, \boldsymbol{m}}^{(k)}(t) y_{1}^{m_{1}} \ldots y_{2 n}{ }^{m_{2 n}}, \\
& f_{j}(\boldsymbol{y}+\boldsymbol{w}, t)-\sum_{i=1}^{2 n} c_{i}(\boldsymbol{y}, t) \frac{\partial}{\partial y_{i}} w_{j}(\boldsymbol{y}, t) \\
& =\sum_{k=1}^{\infty} \varepsilon^{k} \sum_{|\boldsymbol{m}| \leq k+1} g_{j, \boldsymbol{m}}^{(k)}(t) y_{1}^{m_{1}} \ldots y_{2 n}^{m_{2 n}},
\end{aligned}
$$

and by equating the sets of coefficients associated with $y_{1}^{m_{1}}, \ldots, y_{2 n}{ }^{m_{2 n}}$ of the left and right-hand sides of $j$ th component at each order $k$, we obtain the following equation:

$$
\begin{aligned}
& \left(\langle\boldsymbol{\lambda}, \boldsymbol{m}\rangle-\lambda_{j}+\frac{d}{d t}\right) w_{j, \boldsymbol{m}}^{(k)}(t)=g_{j, \boldsymbol{m}}^{(k)}(t)-c_{j, \boldsymbol{m}}^{(k)}(t), \\
& \operatorname{def} \\
& \langle\boldsymbol{\lambda}, \boldsymbol{m}\rangle=\lambda_{1} m_{1}+\cdots+\lambda_{2 n} m_{2 n} .
\end{aligned}
$$

Here at each order $k$ the time-dependent coefficients $w_{j, \boldsymbol{m}}^{(k)}(t)$ and $c_{j, \boldsymbol{m}}^{(k)}(t)$ are unknown while $g_{j, \boldsymbol{m}}^{(k)}(t)$ is known: Since the expansions of $f, c$, and $w$ start with $k=1$ (first order of $\varepsilon$ ), it is seen from Eq. (24) that $g_{j, m}^{(k)}(t)$ only depends on $w_{j, \boldsymbol{m}}^{(1)}(t), \ldots, w_{\boldsymbol{m}}^{(k-1)}(t)$ and $c_{j, \boldsymbol{m}}^{(1)}(t), \ldots, c_{j, \boldsymbol{m}}^{(k-1)}(t)$, that is, the terms of order less than $k$ [For $k=1$, we simply have $\left.g_{j, \boldsymbol{m}}^{(1)}(t)=f_{j, \boldsymbol{m}}^{(1)}(t)\right]$. That is, if $c_{j, \boldsymbol{m}}^{\left(k^{\prime}\right)}(t)$ and $w_{j, \boldsymbol{m}}^{\left(k^{\prime}\right)}(t)$ have been determined in any fashion for $k^{\prime}<k, g_{j, \boldsymbol{m}}^{(k)}(t)$ can be identified uniquely. Therefore, at each order $k$, there successively appear two unknown quantities, $w_{j, \boldsymbol{m}}^{(k)}(t)$ and $c_{j, \boldsymbol{m}}^{(k)}(t)$ with one known quantity $g_{j, m}^{(k)}(t)$. In other words, there is flexibility to establish these two quantities as one wishes.

Here we determine the unknown $w_{j, \boldsymbol{m}}^{(k)}(t)$ so that the equation of motion for $\boldsymbol{y}$ contains as little terms as possible, at each order in $k$ : We can solve Eq. (25) by setting

$$
\begin{aligned}
w_{j, \boldsymbol{m}}^{(k)}(t) & =S\left[\lambda_{j}-\langle\boldsymbol{\lambda}, \boldsymbol{m}\rangle, g_{j, \boldsymbol{m}}^{(k)}\right](t), \\
c_{j, \boldsymbol{m}}^{(k)}(t) & =0,
\end{aligned}
$$

using the same $S$-symbol as in Eqs. (13) and (14). For terms with $m_{j}=1$ and $m_{i}=0(i \neq j)$, we have $\lambda_{j}-\langle\boldsymbol{\lambda}, \boldsymbol{m}\rangle=0$, which prohibits the use of the above solution because $S$ is not well defined for the zero real part [see Eq. (13)]. For such $\boldsymbol{m}$, we must set $w_{j, \boldsymbol{m}}^{(k)}(t)=0$ and $c_{j, \boldsymbol{m}}^{(k)}(t)=g_{j, \boldsymbol{m}}^{(k)}(t)$. Thus the terms proportional to $y_{j}$ remains in the final equation, as shown in Eq. (3). Once $w_{j, \boldsymbol{m}}^{(k)}(t)$ and $c_{j, \boldsymbol{m}}^{(k)}(t)$ are determined with a given $g_{j, \boldsymbol{m}}^{(k)}(t)$ at order $k$, then the results are used to calculate $g_{j, \boldsymbol{m}}^{(k+1)}(t)$ through Eq. (24). With the determined $g_{j, \boldsymbol{m}}^{(k+1)}(t)$, one can further proceed the same procedure to obtain $w_{j, \boldsymbol{m}}^{(k+1)}(t)$ and $c_{j, \boldsymbol{m}}^{(k+1)}(t)$ and so forth.

Once we determine the functions $w_{j}^{(k)}(\boldsymbol{y}, t)$ up to a certain order, the transformation from $\boldsymbol{y}$ to $\boldsymbol{x}$ is given by Eq. (21). The inverse transformation, i.e., $\boldsymbol{y}$ as a function of $\boldsymbol{x}$, can be given by iterative solution of Eq. (21) as follows. Let

$$
\boldsymbol{y}=\boldsymbol{y}^{(k)}(\boldsymbol{x}, t)+O\left(\varepsilon^{k+1}\right)
$$

be the $k$ th order expression of $\boldsymbol{y}$ in terms of $\boldsymbol{x}$. Substituting it into Eq. (21) we have

$$
\begin{aligned}
& y_{j}^{(0)}(\boldsymbol{x}, t)=x_{j}, \\
& y_{j}^{(l+1)}(\boldsymbol{x}, t)=x_{j}-w_{j}\left(\boldsymbol{y}^{(l)}, t\right) \quad(l=0,1, \ldots, k)
\end{aligned}
$$

because $w_{j}(\boldsymbol{y}, t)$ begins with first order of $\varepsilon$.

\section{Equation of motion for the new coordinates}

In Secs. II A-II C we have introduced a framework with which one can determine the coordinate transformation to make the equation of motion into a desired form. One might wish to make all degrees of freedom decoupled in the equation of motion for $\boldsymbol{y}$ as

$$
\dot{y}_{j}=\left[\lambda_{j}+c_{j}(t)\right] y_{j}+O\left(\varepsilon^{N+1}\right)
$$

$(j=1,2, \ldots, 2 n)$. Note that the terms proportional to $y_{j}$ in the equation of $\dot{y}_{j}$ remains as stated in Sec. II C and $c_{j}(t)$ corresponds to $\sum_{k=1}^{N} \varepsilon^{k} c_{j, \boldsymbol{m}}^{(k)}(t)$ with $m_{j}=1$ and $m_{i}=0(i \neq j)$. In this paper, we call the form of Eq. (29) "full NF" in analogy to Hamiltonian systems. The solution is then obtained in an analytical form as

$$
y_{j}(t)=y_{j}\left(t_{0}\right) \exp \left[\int_{t_{0}}^{t}\left(\lambda_{j}+c_{j}\left(t^{\prime}\right)\right) d t^{\prime}\right]+O\left(\varepsilon^{N+1}\right),
$$

where $t_{0}$ is a certain instance of time (the initial condition).

However, if the system has damping oscillatory modes, the full normalization is impossible from the following reason. The complex eigenvalues for the real matrix in Eq. (7) arise in conjugate pair. For example, in the case of uniform friction (see Appendix A), the modes $y_{3}, y_{4}, \ldots$ are the damping oscillatory modes and we have from Eq. (A3) $\operatorname{Re} \lambda_{2 j}=\operatorname{Re} \lambda_{2 j-1} \quad(j=2,3, \ldots, n)$. The combination $m_{2 j-1}=1$ and $m_{i}=0(i \neq 2 j-1)$ will give $\operatorname{Re}\left[\lambda_{2 j}-\langle\boldsymbol{\lambda}, \boldsymbol{m}\rangle\right]=0$. This proof applies not only to the case of uniform friction but also whenever we have oscillatory modes (eigenvalues in conjugate pair). As in Ref. 55, the impossibility of full NF 
can be physically interpreted as resonance between the (damping) oscillatory modes of the system and the external field which has continuous Fourier spectrum (see also Appendix B).

For the purpose of predicting the destination or final state of the reaction, one needs only to know the motion along the unstable direction $y_{1}$, the reaction coordinate decoupled from the others, corresponding to the motion sliding down the barrier into either the reactant or the product regime. Thus we normalize solely the equation of $\dot{y}_{1}$ while leaving other modes as they are,

$$
\begin{aligned}
& \dot{y}_{1}=\left[\lambda_{1}+c_{1}(t)\right] y_{1}+O\left(\varepsilon^{N+1}\right), \\
& \dot{y}_{2}=\lambda_{2} y_{2}+c_{2}(\boldsymbol{y}, t), \\
& \dot{y}_{3}=\lambda_{3} y_{3}+c_{3}(\boldsymbol{y}, t), \\
& \vdots \\
& \dot{y}_{2 n}=\lambda_{2 n} y_{2 n}+c_{2 n}(\boldsymbol{y}, t) .
\end{aligned}
$$

We call this "partial NF." The solution for the motion of $y_{1}$ is given by

$$
y_{1}(t)=y_{1}\left(t_{0}\right) \exp \left[\int_{t_{0}}^{t}\left(\lambda_{1}+c_{1}\left(t^{\prime}\right)\right) d t^{\prime}\right]+O\left(\varepsilon^{N+1}\right) .
$$

Note that the expression for $c_{1}$ begins with first order perturbation term,

$$
c_{1}(t)=\sum_{k=1}^{N} \varepsilon^{k} c_{1}^{(k)}(t)
$$

Of particular importance is the behavior in $t \rightarrow+\infty$. The exponent in Eq. (32) goes to infinity as $t \rightarrow+\infty$ if the long-time average of the perturbative terms in $c_{1}(t)[\mathrm{Eq} .(33)]$ is less than $\lambda_{1}$, that is,

$$
\lim _{t \rightarrow+\infty}\left|\frac{1}{t} \int_{t_{0}}^{t} \sum_{k=1}^{N} \varepsilon^{k} c_{1}^{(k)}\left(t^{\prime}\right) d t^{\prime}\right|<\lambda_{1} .
$$

Since the left-hand side is $O(\varepsilon)$ and the right-hand side is zeroth order, this inequality can usually be expected to hold. As long as this condition is fulfilled,

$$
\lim _{t \rightarrow+\infty} y_{1}(t)=\left\{\begin{array}{cc}
+\infty & \left(y_{1}\left(t_{0}\right)>0\right) \\
-\infty & \left(y_{1}\left(t_{0}\right)<0\right) .
\end{array}\right.
$$

The increase in $\left|y_{1}(t)\right|$ means that the system departs from the vicinity of the saddle point, going either to the product or to the reactant. The most important consequence is that irrespective of the other modes $\left(y_{2}, \ldots, y_{2 n}\right)$, only the knowledge of the sign of $y_{1}$ at any one moment in the region of saddle is sufficient to predict the final state of the reaction, i.e., either ending up at the product or bouncing back to the reactant.

Mathematically, $y_{1}$ and $y_{2}$ correspond, respectively, to the unstable and stable invariant manifolds emanating from the normally hyperbolic invariant manifold ${ }^{57,58}$ in the $y$ space. If one also normalizes the equation for $y_{2}$,

$$
\dot{y}_{2}=\left[\lambda_{2}+c_{2}(t)\right] y_{2}+O\left(\varepsilon^{N+1}\right),
$$

instead of the second line of Eq. (31), similar argument as above will show that the sign of $y_{2}$ can tell us which state the system originated from (without performing any trajectory calculation backward in time or recording the pathway of the trajectory).

\section{E. The physical foundation of reaction under thermal fluctuation}

The transformation from the original coordinates $(\boldsymbol{q}, \dot{\boldsymbol{q}})$ (through $\boldsymbol{u}$ and $\boldsymbol{x}$ ) to $\boldsymbol{y}$ is obtained from Eqs. (8), (11), and (27). In general form, it can be expressed as

$$
\begin{aligned}
y_{1}= & a_{1} q_{1}+a_{2} \dot{q}_{1}-S\left[\lambda_{1}, \widetilde{\xi}_{1}\right](t)+F_{0}[\xi](t) \\
& +\sum_{|\boldsymbol{m}| \geq 2} w_{\boldsymbol{m}} q_{1}^{m_{1}}, \ldots, q_{n}^{m_{n}} \dot{q}_{1}^{m_{n+1}}, \ldots, \dot{q}_{n}^{m_{2 n}} \\
& +\sum_{|\boldsymbol{m}| \geq 1} F_{\boldsymbol{m}}[\boldsymbol{\xi}](t) q_{1}^{m_{1}}, \ldots, q_{n}^{m^{n}} \dot{q}_{1}^{m_{n+1}}, \ldots, \dot{q}_{n}^{m_{2 n}} .
\end{aligned}
$$

This provides us with the firm physical foundation of why the reaction takes place in terms of the components that appeared in Eq. (37) that can be classified into the following five distinct components.

(i) The linear terms in the original position $q_{1}$ and velocity $\dot{q}_{1}$ where the coefficients $a_{1}$ and $a_{2}$ are determined by the linear transformation by Eq. (8), reflecting the harmonic part of the potential and friction constants.

(ii) $S\left[\lambda_{1}, \tilde{\xi}_{1}\right](t)$, linear term in the random force $\widetilde{\xi}_{1}(t)$, is interpreted as the motion directly excited by the kick from the environment along the reactive direction.

(iii) The nonlinear terms with coefficients $w_{\boldsymbol{m}}$ in $\boldsymbol{q}$ and $\dot{\boldsymbol{q}}$, containing no $\boldsymbol{\xi}(t)$, describe the driving force of the reaction arising from nonlinear couplings that are regarded as intrinsic to the reacting system.

(iv) The term $F_{0}[\xi](t)$ is a nonlinear functional of the random force $\widetilde{\xi}_{i}(t)$ along all the directions $(i=1, \ldots, n)$ but not containing $\boldsymbol{q}$ or $\dot{\boldsymbol{q}}$. This term arises only when there exist both $\boldsymbol{\xi}(t)$ and the nonlinearity in the potential $\boldsymbol{U}$. For example, when the vibrational motion along the nonreactive mode is excited by a kick from the environment, this motion then changes the reactivity through nonlinear couplings between the nonreactive and the reactive modes.

(v) The terms with the time-dependent coefficient $F_{m}[\xi](t)$ can be called "environment-mediated coupling" since the amplitude of couplings among $(\boldsymbol{q}, \dot{\boldsymbol{q}})$ depends on the $\boldsymbol{\xi}(t)$. That is, the force from the environment disturbs the position of the system on the landscape of potential, resulting in the change of the coupling strength.

Note here that the coefficients $w_{\boldsymbol{m}}, F_{0}[\boldsymbol{\xi}](t)$, and $F_{m}[\xi](t)$, obtained by the nonlinear transformation in Sec. II C, generally depend on the linear eigenvalues $\boldsymbol{\lambda}$ (and therefore on the friction constants) through Eq. (26). The striking observation from $F_{0}[\xi](t)$ and $F_{m}[\xi](t)$ is that the 
nonlinearity intrinsic to the system (e.g., solute), and the random force and friction exerted by the environment (e.g., solvent) influence the reaction not only in separate ways, but in cooperation with each other. The effect cannot be simply classified as environment or nonlinear effect, but it should be understood as a "combination" of them.

Insofar, under some moderate conditions [e.g., Eq. (34)], it has been shown that there exists a reaction coordinate $y_{1}$ whose sign, in principle, determines the fate of the reaction. The transformation from the original coordinates to $y_{1}$ depends on each instance of the stochastic forces $\xi_{j}(t)$. Theoretically, if one knew the time series of $\boldsymbol{\xi}(t)$ for all $t$ in advance, one could precisely predict the fate of the reaction by the sign of $y_{1}$ within the order of perturbation. Equation (13) also requires all the future values of $\widetilde{\xi}_{j}$ for Re $\lambda_{1}>0$ and all the past values for $\operatorname{Re} \lambda_{j}<0$. In realistic situations, the system cannot "know" the time evolution of $\boldsymbol{\xi}(t)$ for each instance in advance. What one may assume is the characteristic properties of random forces as "an ensemble" rather than a single instance.

As seen in Sec. II C, the analytical expressions for $y_{1}$ contains combinations of stochastic forces $\xi_{j}(t)$ "wrapped" by $S$-functionals [Eq. (13)]. Assuming the statistical properties of $\xi_{j}$, i.e., $\left\langle\xi_{j}\right\rangle=0$ and the fluctuation-dissipation theorem given by Eq. (2), the ensemble average of several products and convolutions of $S$-functionals taken over all realizations of stochastic random force can be calculated analytically,

$$
\begin{aligned}
& \left\langle S\left[\lambda_{i}, \xi_{j}\right]\right\rangle=0, \\
& \left\langle S\left[\lambda_{1}, \xi_{i}\right] S\left[\lambda_{2}, \xi_{j}\right]\right\rangle=2 k_{\mathrm{B}} T \gamma_{i j} F\left(\lambda_{1}, \lambda_{2}\right), \\
& \left\langle S\left[\lambda_{3}, S\left[\lambda_{1}, \xi_{i}\right] S\left[\lambda_{2}, \xi_{j}\right]\right]\right\rangle=-2 k_{\mathrm{B}} T \lambda_{3}^{-1} \gamma_{i j} F\left(\lambda_{1}, \lambda_{2}\right),
\end{aligned}
$$

with the symbol $F$ defined by

$$
\begin{aligned}
& F\left(\lambda_{1}, \lambda_{2}\right) \\
& = \begin{cases}\frac{1}{\lambda_{1}+\lambda_{2}} & \text { (if } \left.\operatorname{Re} \lambda_{1}>0 \text { and } \operatorname{Re} \lambda_{2}>0\right) \\
-\frac{1}{\lambda_{1}+\lambda_{2}} & \text { (if } \left.\operatorname{Re} \lambda_{1}<0 \text { and } \operatorname{Re} \lambda_{2}<0\right) \\
0 & \text { (otherwise). }\end{cases}
\end{aligned}
$$

Some formulas arising in higher orders are also presented in Appendix C.

Thanks to these formulas using the statistical properties of $\xi_{i}(t)$, one can look into the question of how the dynamical structure persists under thermal fluctuation without knowing all $\boldsymbol{\xi}(t)$ in advance. As a function of initial conditions on the position-velocity space $(\boldsymbol{q}, \dot{\boldsymbol{q}})$ of the system, one can a priori capture the reaction probability that is given by the probability of $y_{1}>0$ even under the stochastic nature of $\xi_{j}(t)$ exerted by the environment (without performing any trajectory calculation). The dividing hypersurface to separate the positionvelocity space into the two disjoint regions, i.e., mainly reactive and mainly nonreactive regions, under thermal fluctuation can be defined by $\left\langle y_{1}\right\rangle=0\left(P_{\text {fold }}\right.$ in the words of protein folding community). How and to what extent the dynamical structure survives under the fluctuating environment can be investigated by analyzing the statistical distribution of $y_{1}$ including the analytical formulation of $P_{\text {fold }}$ and their dependence on $\boldsymbol{q}, \dot{\boldsymbol{q}}$, temperature, and so forth.

The ensemble average of $y_{1}$ with respect to thermal noise, $\left\langle y_{1}\right\rangle$, is given by

$$
\begin{aligned}
\left\langle y_{1}\right\rangle= & a_{1} q_{1}+a_{2} \dot{q}_{1}+\bar{F}_{0}\left(k_{\mathrm{B}} T\right) \\
& +\sum_{|\boldsymbol{m}| \geq 2} w_{\boldsymbol{m}} q_{1}{ }^{m_{1}}, \ldots, q_{n}{ }^{m_{n}} \dot{q}_{1}^{m_{n+1}}, \ldots, \dot{q}_{n}^{m_{2 n}} \\
& +\sum_{|\boldsymbol{m}| \geq 1} \bar{F}_{\boldsymbol{m}}\left(k_{\mathrm{B}} T\right) q_{1}{ }^{m_{1}}, \ldots, q_{n}{ }^{{ }_{n}} \dot{q}_{1}^{m_{n+1}}, \ldots, \dot{q}_{n}^{m_{2 n}},
\end{aligned}
$$

where $\bar{F}_{0}\left(k_{\mathrm{B}} T\right)$ and $\bar{F}_{m}\left(k_{\mathrm{B}} T\right)$ are the averages of the corresponding terms in Eq. (37) and are functions of temperature. The direct solvent effect $S\left[\lambda_{1}, \widetilde{\xi}_{1}\right](t)$ in Eq. (37) vanishes in Eq. (40). This term only results in the fluctuation of $y_{1}$ around the average and does not provide any information concerning the persistence of the dynamical structure of the system against thermal noise. The terms with $F_{0}$ and $F_{m}$ describing the "nonlinear cooperation" between the system and the environment and those with $w_{m}$ arising from the nonlinearity intrinsic to the system are essential for understanding the mechanism of the robustness of reaction under thermal fluctuation. Note here that the direct solvent effect $S\left[\lambda_{1}, \widetilde{\xi}_{1}\right](t)$ vanishes under the average over all realizations of $\boldsymbol{\xi}(t)$ and all of terms with $F_{0}, F_{\boldsymbol{m}}$, and $w_{\boldsymbol{m}}$ cannot be obtained within the framework of linear approximation. ${ }^{44,51-53}$

\section{F. Nonlinear resonances under thermal fluctuation}

We discuss the possible scenario of the breakdown of the form of Eq. (32), which makes reaction totally unpredictable, implying that the reaction undergoes only in a purely stochastic fashion.

When some combination of $\boldsymbol{m}$ [other than $m_{j}=1$ and $\left.m_{i}=0(i \neq j)\right]$ causes zero (or very small) real part in Eq. (26), the NF calculation cannot be carried out as in Sec. II C. This is closely related to the well-known resonance problem in dynamical systems. Judging the existence of such resonances requires a knowledge of the specific values of the eigenvalues $\lambda_{1}, \ldots, \lambda_{2 n}$, and therefore depends on the system. Here let us consider the case of uniform friction $\Gamma=\gamma \mathbf{1}$ (see also Appendix A). The resonance condition to break the form of the equation of motion for $y_{1}$ [Eq. (31)] becomes

$$
\begin{aligned}
\operatorname{Re}\left(\lambda_{1}-\langle\lambda, m\rangle\right)= & -\left(m_{1}-1\right) \lambda_{1}+m_{2}\left|\lambda_{2}\right| \\
& +\left(m_{3}+\cdots+m_{2 n}\right) \gamma / 2 \approx 0 .
\end{aligned}
$$

The first term in the right-hand side is negative, while the second and the third terms are positive. Therefore the first term may cancel with either the second or the third, or both. In the cases where the first and the second terms cancel with each other, it might not be appropriate to call this effect "resonance" because both $\lambda_{1}$ and $\lambda_{2}$ arise from the reactive mode. This situation may cause the change in dynamical structure of the reactive mode through the appearance of the terms of the type $\left(y_{1}^{m_{1}} y_{2}^{m_{2}}\right) y_{1}$ with $m_{1}: m_{2} \approx\left|\lambda_{2}\right|: \lambda_{1}$ in the 
equation of motion for $y_{1}$. The cancellation of the first term with the third is what is unknown in Hamiltonian systems. In isolated systems, eigenvalues of the nonreactive modes are purely imaginary. There the resonance does not occur between the reactive mode and the nonreactive modes, supporting the separability of the reactive mode..$^{8,10,16,17,32,59}$ In condensed phase, however, the reactive mode and the nonreactive ones can be in "resonance" through the appearance of $-\gamma / 2$ in the nonreactive mode eigenvalues. This may break the separability and make the reaction coordinate essentially multidimensional. Appearance of purely stochastic process may thus be explained through this picture.

It should also be pointed out that breakdown of the condition of Eq. (34) may lead to the change in dynamical stability near the saddle point such as bifurcation of no-return transition state in many degrees of freedom Hamiltonian systems. ${ }^{26}$ That is, the system will remain in the region of saddle for a certain duration without falling into the asymptotic regions. The time-dependent coefficient of $y_{1}$ arises primarily from the term with $m_{1}=1, m_{2}=\cdots=m_{2 n}$ $=0$ in Eq. (12), which in turn originates from the introduction of the time-dependent shift [Eq. (11)]. More precisely, terms proportional in $x_{1}$ appears when the shift [Eq. (11)] is substituted in the nonlinear terms in the equation of motion for $\boldsymbol{u}$ [Eq. (9)]. Thus the higher-order correlations among the random forces $\boldsymbol{\xi}(t)$ may stabilize the equilibrium point if the effect of nonlinearity is sufficiently large.

\section{SUMMARY AND OUTLOOK}

Based on multidimensional underdamped Langevin equation for a wide class of reacting systems of many degrees of freedom (i.e., without any a priori assumption about the dimension or the form of the potential of mean force), a theory has been presented to single out a new reaction coordinate (called $y_{1}$ in this paper) decoupled from the others under thermal fluctuation. The reaction coordinate is an analytical functional of the position coordinates $\boldsymbol{q}$ and the velocities $\dot{\boldsymbol{q}}$ of the system, and the random force $\boldsymbol{\xi}(t)$ exerted by the environment. The sign of the coordinate at any instantaneous moment in the region of saddle can, in principle, determine the fate of the reaction, i.e., whether the reaction system will proceed to the products or go back to the reactants.

The analytical expression of the reaction coordinate enables us to decompose the origin of reaction into the following five components: (i) linear terms in the original position coordinate $q_{1}$ and velocity $\dot{q}_{1}$ along the reactive normal mode coordinate, which reflect the harmonic part of the potential and friction constants; (ii) time-dependent linear term in the random force $\xi_{1}(t)$ acting along $q_{1}$. This is a transition state trajectory introduced by Bartsch et al. ${ }^{51-53}$ This implies the motion caused directly by a kick from the solvent; (iii) nonlinear terms in the original position $\boldsymbol{q}$ and the velocity $\dot{\boldsymbol{q}}$, but not containing $\boldsymbol{\xi}(t)$. These are the effects of nonlinear couplings that are intrinsic to the system since they appear even in the absence of the random force; (iv) second and higherorder terms in $\boldsymbol{\xi}(t)$, but containing neither $\boldsymbol{q}$ nor $\dot{\boldsymbol{q}}$; these terms, for example, provide a shift of the border of the reac- tion caused by the vibrational excitation of the nonreactive mode by a kick from the solvent, followed by nonlinear couplings between the nonreactive and the reactive modes; (v) nonlinear terms including both $\boldsymbol{\xi}(t)$ and $(\boldsymbol{q}, \dot{\boldsymbol{q}})$. These are what can be called "environment-mediated couplings" since the amplitude of couplings among $(\boldsymbol{q}, \dot{\boldsymbol{q}})$ depends on the $\boldsymbol{\xi}(t)$ or temperature of the environment. That is, the force from the environment disturbs the position of the system on the potential surface, resulting in the change in the coupling strength. (iv) and (v) are both regarded as the effects of nonlinear cooperation among the nonlinearities inherent to the system and the thermal fluctuations $\boldsymbol{\xi}(t)$.

Based on the statistical properties of random force, one can know a priori a well-defined boundary of the reaction, as an analytical nonlinear function of position coordinates $\boldsymbol{q}$ and velocities $\dot{\boldsymbol{q}}$ with friction constants and temperature $T$, which divides the position-velocity space in the region of saddle into mainly reactive and mainly nonreactive regions under thermal fluctuation. Component (ii), the direct solvent effect $S\left[\lambda_{1}, \widetilde{\xi}_{1}\right](t),{ }^{51-53}$ has the same probability for both signs in $y_{1}$ and vanishes under the ensemble of $\widetilde{\xi}_{1}(t)$. Components (iii)-(v) derived in this paper should play a pivotal role in unveiling how the dynamical structure of the system (e.g., proteins) arising from nonlinear interactions among different modes under the influence of stochastic, thermal fluctuation. Our preliminary calculation using the model system showed, as one may anticipate, that the more the friction constants increases, the more the coefficients of homogeneous polynomials at each order involving velocities $\dot{\boldsymbol{q}}$ tends to decrease in magnitude. In other words, our theory can naturally capture what size of friction constants exerted by the environment make the effect of moment of inertia negligible (rather than postulating the overdamped Langevin formulation). Note however that this does not necessarily imply the cease of all components (iii)-(v). There exist yet the nonlinear terms that are intrinsic to system (iii) and the combined effects between nonlinearity and the surrounding environment [(iv) and (v)]. "Dynamical structure" we have termed in this paper is regarded as a dynamic entity composed of nonlinearity inherent to the system (which may involve both the position coordinates and velocities) and their cooperation with the thermal fluctuation.

We have discussed the possible scenario which makes reaction totally unpredictable. The violation of the condition of Eq. (34) makes the stability change in the region of rankone saddle although it unlikely happens as long as the system resides within the (asymptotic) convergence radius of the perturbation. Like in the theory for isolated systems, the breakdown of the perturbation theory can be understood in terms of resonance. In isolated systems, only one mode has a real eigenvalue, with others being purely imaginary in the region of rank-one saddle. There resonance does not occur between the reactive mode and all the other nonreactive modes, supporting the separability of the reactive mode. ${ }^{8,10,16,17,32,59}$ In the presence of friction, however, all the eigenvalues have nonzero real part arising from dissipation so that a new type of resonance may emerge. It would be interesting to seek for the mechanism of the change in dy- 
namical structure or the transition to a purely stochastic reaction in terms of these new types of resonance in the region of rank-one saddles in dissipative systems.

\section{ACKNOWLEDGMENTS}

We thank Professor Turgay Uzer and Professor Thomas Bartsch for letting us know their researches in advance, and Professor Mikito Toda for stimulating discussions. This work was supported by the Research Fellowships of the Japan Society for the Promotion of Science for Young Scientists (to S.K.) and by JSPS, JST/CREST, and Priority Area "Molecular Theory for Real Systems" (to T.K.). S.K. is a research fellow of the Japan Society for the Promotion of Science.

\section{APPENDIX A: LINEAR FREQUENCIES FOR UNIFORM FRICTION}

The eigenvalues are obtained analytically for the case of uniform friction $\boldsymbol{\Gamma}=\gamma \mathbf{1} .^{53}$ There is one positive value,

$$
\lambda_{1}=\frac{1}{2}\left(-\gamma+\sqrt{\gamma^{2}+4 k^{2}}\right)>0,
$$

and one negative value,

$$
\lambda_{2}=\frac{1}{2}\left(-\gamma-\sqrt{\gamma^{2}+4 k^{2}}\right)<0,
$$

both corresponding to the reactive direction $q_{1}$, where $k$ is the curvature along the reactive coordinate. Other directions correspond to the nonreactive modes, and we have for their eigenvalues

$$
\begin{aligned}
& \lambda_{2 j-1}=\frac{1}{2}\left(-\gamma+\mathrm{i} \sqrt{4 \omega_{j}^{2}-\gamma^{2}}\right), \\
& \lambda_{2 j}=\frac{1}{2}\left(-\gamma-\mathrm{i} \sqrt{4 \omega_{j}^{2}-\gamma^{2}}\right), \\
& (j=2,3, \ldots, n),
\end{aligned}
$$

where $\omega_{j}$ is the frequencies of the nonreactive mode without friction. If $\gamma<2 \omega_{j}$, these are complex values with negative real parts, corresponding to damping oscillatory motions. Note that they appear in conjugate pair. In the case of large friction $\left(\gamma>2 \omega_{j}\right)$, these values become negative real, corresponding to simply damping modes.

\section{APPENDIX B: ON THE S-SYMBOL WITH PURELY IMAGINARY ARGUMENTS}

In Eq. (13), the $S$-symbol is defined for positive and negative real parts separately. That is, we have not given any definition for $S[\mu, \psi]$ when the argument $\mu$ has zero real part. As have been done in Ref. 55, one could define it by adding a small real part,

$$
\begin{aligned}
S_{-}[\mathrm{i} \omega, \psi](t) & =\lim _{\varepsilon \rightarrow+0} S[-\varepsilon+\mathrm{i} \omega, \psi](t) \\
& =\lim _{\varepsilon \rightarrow+0} \int_{-\infty}^{0} \exp (-(-\varepsilon+\mathrm{i} \omega) \tau) \psi(t+\tau) d \tau
\end{aligned}
$$

or

$$
\begin{aligned}
S_{+}[\mathrm{i} \omega, \psi](t) & =\lim _{\varepsilon \rightarrow+0} S[\varepsilon+\mathrm{i} \omega, \psi](t) \\
& =\lim _{\varepsilon \rightarrow+0}-\int_{0}^{+\infty} \exp (-(\varepsilon+\mathrm{i} \omega) \tau) \psi(t+\tau) d \tau .
\end{aligned}
$$

Any linear combination of these will qualify as the definition of $S[\mathrm{i} \omega, \psi](t)$ since it satisfies Eq. (14). However, for the case that the function $\psi(t)$ includes a white noise, these integrations do not converge, or more precisely, give a variable whose variance is infinity since the integrands do not decay as $t \rightarrow \pm \infty$ for $\varepsilon=0$. Since the above equations have the form of Fourier transform of $\psi$, their divergence can physically be interpreted as resonance between the frequency of the system and the external force. In the present paper, the random force is a white noise, and therefore contains all frequency components. In the case of colored noise, it may happen that some Fourier component of the external force becomes zero, turning off the resonance. In general, however, the resonance is expected to occur since the time-dependent force has a continuous spectrum in a wide range of frequency.

APPENDIX C: ENSEMBLE AVERAGES OF SOME EXPRESSIONS APPEARING IN HIGH ORDER

$$
\begin{aligned}
&\left\langle S\left[\lambda_{1}, \xi_{i}\right] S\left[\lambda_{2}, \xi_{j}\right] S\left[\lambda_{3}, \xi_{k}\right] S\left[\lambda_{4}, \xi_{\ell}\right]\right\rangle=\left(2 k_{\mathrm{B}} T\right)^{2}\left\{\gamma_{i j} \gamma_{k \ell} F\left(\lambda_{1}, \lambda_{2}\right) F\left(\lambda_{3}, \lambda_{4}\right)+\gamma_{i k} \gamma_{j \ell} F\left(\lambda_{1}, \lambda_{3}\right) F\left(\lambda_{2}, \lambda_{4}\right)+\gamma_{i \ell} \gamma_{j k} F\left(\lambda_{1}, \lambda_{4}\right) F\left(\lambda_{2}, \lambda_{3}\right)\right\}, \\
&\left\langle S\left[\lambda_{1}, \xi_{i}\right] S\left[\lambda_{2}, \xi_{j}\right] S\left[\lambda_{5}, S\left[\lambda_{3}, \xi_{k}\right] S\left[\lambda_{4}, \xi_{\ell}\right]\right]\right\rangle=\left(2 k_{\mathrm{B}} T\right)^{2}\left[-\frac{1}{\lambda_{5}} \gamma_{i j} \gamma_{k \ell} F\left(\lambda_{1}, \lambda_{2}\right) F\left(\lambda_{3}, \lambda_{4}\right)+\frac{\gamma_{i k} \gamma_{j \ell}}{\left(\lambda_{1}+\lambda_{3}\right)\left(\lambda_{2}+\lambda_{4}\right)}\right. \\
& \times\left\{M\left(\lambda_{1}, \lambda_{2}, \lambda_{5}\right)+M\left(-\lambda_{3},-\lambda_{4}, \lambda_{5}\right)+M\left(\lambda_{4},-\lambda_{1},-\lambda_{5}\right)+M\left(\lambda_{3},-\lambda_{2},-\lambda_{5}\right)\right\} \\
&+\frac{\gamma_{i \ell} \gamma_{j k}}{\left(\lambda_{1}+\lambda_{4}\right)\left(\lambda_{2}+\lambda_{3}\right)}\left\{M\left(\lambda_{1}, \lambda_{2}, \lambda_{5}\right)+M\left(-\lambda_{3},-\lambda_{4}, \lambda_{5}\right)+M\left(\lambda_{3},-\lambda_{1},-\lambda_{5}\right)\right. \\
&\left.\left.+M\left(\lambda_{4},-\lambda_{2},-\lambda_{5}\right)\right\}\right]
\end{aligned}
$$




$$
\begin{aligned}
\left\langle S\left[\lambda_{1}, \xi_{i}\right] S\left[\lambda_{5}, S\left[\lambda_{2}, \xi_{j}\right] S\left[\lambda_{3}, \xi_{k}\right] S\left[\lambda_{4}, \xi_{\ell}\right]\right]\right\rangle= & \left(2 k_{\mathrm{B}} T\right)^{2}\left[\frac{\gamma_{i j} \gamma_{k \ell}}{\lambda_{1}+\lambda_{2}}\left\{F\left(-\lambda_{2}, \lambda_{5}\right)-F\left(\lambda_{1}, \lambda_{5}\right)\right\} F\left(\lambda_{3}, \lambda_{4}\right)+\frac{\gamma_{i k} \gamma_{j \ell}}{\lambda_{1}+\lambda_{3}}\left\{F\left(-\lambda_{3}, \lambda_{5}\right)\right.\right. \\
& \left.\left.-F\left(\lambda_{1}, \lambda_{5}\right)\right\} F\left(\lambda_{2}, \lambda_{4}\right)+\frac{\gamma_{i \ell} \gamma_{j k}}{\lambda_{1}+\lambda_{4}}\left\{F\left(-\lambda_{4}, \lambda_{5}\right)-F\left(\lambda_{1}, \lambda_{5}\right)\right\} F\left(\lambda_{2}, \lambda_{3}\right)\right],
\end{aligned}
$$

$$
\begin{aligned}
\left\langle S\left[\lambda_{5}, S\left[\lambda_{1}, \xi_{i}\right] S\left[\lambda_{2}, \xi_{j}\right]\right] S\left[\lambda_{6}, S\left[\lambda_{3}, \xi_{k}\right] S\left[\lambda_{4}, \xi_{\ell}\right]\right]\right\rangle= & \left(2 k_{\mathrm{B}} T\right)^{2}\left[\frac{\gamma_{i j} \gamma_{k \ell}}{\lambda_{5} \lambda_{6}} F\left(\lambda_{1}, \lambda_{2}\right) F\left(\lambda_{3}, \lambda_{4}\right)+\frac{\gamma_{i k} \gamma_{j \ell}}{\left(\lambda_{1}+\lambda_{3}\right)\left(\lambda_{2}+\lambda_{4}\right)\left(\lambda_{5}+\lambda_{6}\right)}\right. \\
& \times\left\{M\left(\lambda_{1}, \lambda_{2},-\lambda_{5}\right)-M\left(\lambda_{1}, \lambda_{2}, \lambda_{6}\right)-M\left(\lambda_{1},-\lambda_{4},-\lambda_{5}\right)+M\left(\lambda_{1},-\lambda_{4}, \lambda_{6}\right)\right. \\
& -M\left(-\lambda_{3}, \lambda_{2},-\lambda_{5}\right)+M\left(-\lambda_{3}, \lambda_{2}, \lambda_{6}\right)+M\left(-\lambda_{3},-\lambda_{4},-\lambda_{5}\right)-M\left(-\lambda_{3},\right. \\
& \left.\left.-\lambda_{4}, \lambda_{6}\right)\right\}+\frac{\gamma_{i \ell} \gamma_{j k}}{\left(\lambda_{1}+\lambda_{4}\right)\left(\lambda_{2}+\lambda_{3}\right)\left(\lambda_{5}+\lambda_{6}\right)} \times\left\{M\left(\lambda_{1}, \lambda_{2},-\lambda_{5}\right)-M\left(\lambda_{1}, \lambda_{2}, \lambda_{6}\right)\right. \\
& -M\left(\lambda_{1},-\lambda_{3},-\lambda_{5}\right)+M\left(\lambda_{1},-\lambda_{3}, \lambda_{6}\right)-M\left(-\lambda_{4}, \lambda_{2},-\lambda_{5}\right)+M\left(-\lambda_{4}, \lambda_{2}, \lambda_{6}\right) \\
& \left.\left.+M\left(-\lambda_{4},-\lambda_{3},-\lambda_{5}\right)-M\left(-\lambda_{4},-\lambda_{3}, \lambda_{6}\right)\right\}\right],
\end{aligned}
$$

$$
\begin{aligned}
\left\langle S\left[\lambda_{6}, S\left[\lambda_{5}, S\left[\lambda_{1}, \xi_{i}\right] S\left[\lambda_{2}, \xi_{j}\right]\right] S\left[\lambda_{3}, \xi_{k}\right]\right] S\left[\lambda_{4}, \xi_{\ell}\right]\right\rangle= & \left(2 k_{\mathrm{B}} T\right)^{2}\left[\frac{\gamma_{i j} \gamma_{k \ell}}{\lambda_{5}\left(\lambda_{3}-\lambda_{6}\right)} F\left(\lambda_{1}, \lambda_{2}\right)\left\{F\left(\lambda_{4}, \lambda_{6}\right)-F\left(\lambda_{3}, \lambda_{4}\right)\right\}+\frac{\gamma_{i k} \gamma_{j \ell}}{\left(\lambda_{1}+\lambda_{3}\right)\left(\lambda_{2}+\lambda_{4}\right)}\right. \\
& \times\left\{\frac { 1 } { - \lambda _ { 6 } + \lambda _ { 2 } } \left(M\left(\lambda_{1}, \lambda_{6},-\lambda_{5}\right)-M\left(\lambda_{1}, \lambda_{2},-\lambda_{5}\right)-M\left(-\lambda_{3}, \lambda_{6},-\lambda_{5}\right)\right.\right.
\end{aligned}
$$$$
\left.+M\left(-\lambda_{3}, \lambda_{2},-\lambda_{5}\right)\right)-\frac{1}{\lambda_{6}+\lambda_{4}}\left(-M\left(\lambda_{1}, \lambda_{6},-\lambda_{5}\right)+M\left(\lambda_{1},-\lambda_{4},-\lambda_{5}\right)\right.
$$$$
\left.\left.+M\left(-\lambda_{3}, \lambda_{6},-\lambda_{5}\right)-M\left(\lambda_{3}, \lambda_{4}, \lambda_{5}\right)\right)\right\}+\frac{\gamma_{i \ell} \gamma_{j k}}{\left(\lambda_{2}+\lambda_{3}\right)\left(\lambda_{1}+\lambda_{4}\right)}
$$$$
\times\left\{\frac { 1 } { - \lambda _ { 6 } + \lambda _ { 1 } } \left(M\left(\lambda_{2}, \lambda_{6},-\lambda_{5}\right)-M\left(\lambda_{1}, \lambda_{2},-\lambda_{5}\right)-M\left(-\lambda_{3}, \lambda_{6},-\lambda_{5}\right)\right.\right.
$$$$
\left.+M\left(-\lambda_{3}, \lambda_{1},-\lambda_{5}\right)\right)-\frac{1}{\lambda_{6}+\lambda_{4}}\left(-M\left(\lambda_{2}, \lambda_{6},-\lambda_{5}\right)+M\left(\lambda_{2},-\lambda_{4},-\lambda_{5}\right)\right.
$$$$
\left.\left.\left.+M\left(-\lambda_{3}, \lambda_{6},-\lambda_{5}\right)-M\left(\lambda_{3}, \lambda_{4}, \lambda_{5}\right)\right)\right\}\right] \text {, }
$$

with the symbol $M$ defined by

$$
M\left(\lambda_{1}, \lambda_{2}, \lambda_{3}\right)= \begin{cases}-\frac{1}{\lambda_{1}+\lambda_{2}+\lambda_{2}} & \left(\text { if } \operatorname{Re} \lambda_{1}>0, \operatorname{Re} \lambda_{2}>0, \operatorname{Re} \lambda_{3}>0, \text { or } \operatorname{Re} \lambda_{1}<0, \operatorname{Re} \lambda_{2}<0, \operatorname{Re} \lambda_{3}<0\right) \\ 0 & \text { (otherwise) }\end{cases}
$$

Terms with odd number of $\xi_{j}$ has zero average due to the symmetry.

${ }^{1}$ H. A. Kramers, Physica (Amsterdam) 7, 284 (1940).

${ }^{2}$ R. F. Grote and J. T. Hynes, J. Chem. Phys. 73, 2715 (1980).

${ }^{3}$ H. Mori, Prog. Theor. Phys. 33, 423 (1965).

${ }^{4}$ R. Zwanzig, J. Stat. Phys. 9, 215 (1973).

${ }^{5}$ G. van der Zwan and J. T. Hynes, J. Chem. Phys. 78, 4174 (1983).

${ }^{6}$ M. Tokuyama and H. Mori, Prog. Theor. Phys. 55, 411 (1976).

${ }^{7}$ A. Nitzan, P. Ortoleva, J. Deutch, and J. Ross, J. Chem. Phys. 61, 1056 (1974).

${ }^{8}$ T. Komatsuzaki and M. Nagaoka, J. Chem. Phys. 105, 10838 (1996).
${ }^{9}$ T. Komatsuzaki and M. Nagaoka, Chem. Phys. Lett. 265, 91 (1997).

${ }^{10}$ T. Komatsuzaki and R. S. Berry, J. Chem. Phys. 110, 9160 (1999).

${ }^{11}$ T. Komatsuzaki and R. S. Berry, Phys. Chem. Chem. Phys. 1, 1387 (1999).

${ }^{12}$ T. Komatsuzaki and R. S. Berry, J. Chem. Phys. 115, 4105 (2001).

${ }^{13}$ T. Komatsuzaki and R. S. Berry, J. Mol. Struct.: THEOCHEM 506, 55 (2000).

${ }^{14}$ T. Komatsuzaki and R. S. Berry, Proc. Natl. Acad. Sci. U.S.A. 98, 7666 (2001).

${ }^{15}$ T. Komatsuzaki and R. S. Berry, J. Phys. Chem. A 106, 10945 (2002).

${ }^{16} \mathrm{~S}$. Wiggins, L. Wiesenfeld, C. Jaffé, and T. Uzer, Phys. Rev. Lett. 86, $5478(2001)$.

${ }^{17}$ T. Uzer, C. Jaffé, J. Palacián, P. Yanguas, and S. Wiggins, Nonlinearity 
15, 957 (2002)

${ }^{18}$ H. Waalkens, A. Burbanks, and S. Wiggins, J. Chem. Phys. 121, 6207 (2004).

${ }^{19}$ H. Waalkens, A. Burbanks, and S. Wiggins, J. Phys. A 37, L257 (2004).

${ }^{20}$ H. Waalkens and S. Wiggins, J. Phys. A 37, L435 (2004).

${ }^{21}$ S. Kawai, Y. Fujimura, O. Kajimoto, T. Yamashita, C.-B. Li, T. Komatsuzaki, and M. Toda, Phys. Rev. A 75, 022714 (2007).

${ }^{22}$ S. Kawai, C. Jaffé, and T. Uzer, J. Phys. B 38, S261 (2005).

${ }^{23}$ C.-B. Li, Y. Matsunaga, M. Toda, and T. Komatsuzaki, J. Chem. Phys. 123, 184301 (2005).

${ }^{24}$ C.-B. Li, A. Shojiguchi, M. Toda, and T. Komatsuzaki, Few-Body Syst. 38, 173 (2006).

${ }^{25}$ C.-B. Li, A. Shojiguchi, M. Toda, and T. Komatsuzaki, Phys. Rev. Lett. 97, 028302 (2006).

${ }^{26}$ C.-B. Li, M. Toda, and T. Komatsuzaki, J. Chem. Phys. 130, 124116 (2009).

${ }^{27}$ E. R. Lovejoy, S. K. Kim, and C. B. Moore, Science 256, 1541 (1992).

${ }^{28}$ E. R. Lovejoy and C. B. Moore, J. Chem. Phys. 98, 7846 (1993).

${ }^{29}$ W. H. Miller, Faraday Discuss. Chem. Soc. 62, 40 (1977).

${ }^{30}$ T. Seideman and W. H. Miller, J. Chem. Phys. 95, 1768 (1991).

${ }^{31}$ S. Keshavamurthy and W. H. Miller, Chem. Phys. Lett. 205, 96 (1993).

${ }^{32}$ R. Hernandez and W. H. Miller, Chem. Phys. Lett. 214, 129 (1993).

${ }^{33}$ R. Hernandez, J. Chem. Phys. 101, 9534 (1994).

${ }^{34}$ R. A. Marcus, Science 256, 1523 (1992).

${ }^{35}$ D. J. Wales and R. S. Berry, J. Phys. B 24, L351 (1991).

${ }^{36}$ R. J. Hinde, R. S. Berry, and D. J. Wales, J. Chem. Phys. 96, 1376 (1992).

${ }^{37}$ C. Amitrano and R. S. Berry, Phys. Rev. Lett. 68, 729 (1992).

${ }^{38}$ R. J. Hinde and R. S. Berry, J. Chem. Phys. 99, 2942 (1993).

${ }^{39}$ T. Komatsuzaki and R. S. Berry, Adv. Chem. Phys. 123, 79 (2002).

${ }^{40}$ M. Toda, Adv. Chem. Phys. 123, 153 (2002).

${ }^{41}$ T. Komatsuzaki and R. S. Berry, Adv. Chem. Phys. 130, 143 (2005).

${ }^{42}$ C. Jaffé, S. Kawai, J. Palacián, P. Yanguas, and T. Uzer, Adv. Chem. Phys. 130, 171 (2005).
${ }^{43}$ M. Toda, Adv. Chem. Phys. 130, 337 (2005).

${ }^{44}$ T. Bartsch, J. M. Moix, R. Hernandez, S. Kawai, and T. Uzer, Adv. Chem. Phys. 140, 191 (2008).

${ }^{45}$ H. Waalkens, R. Schubert, and S. Wiggins, Nonlinearity 21, R1 (2008).

${ }^{46}$ S. Kawai, H. Teramoto, C.-B. Li, T. Komatsuzaki, and M. Toda, "Dynamic reaction theory based on geometric structures in phase space," Adv. Chem. Phys. (in press).

${ }^{47}$ Geometrical Structures of Phase Space in Multidimensional Chaos: Applications to Chemical Reaction Dynamics in Complex Systems, Advances in Chemical Physics, edited by M. Toda, T. Komatsuzaki, T. Konishi, R. S. Berry, and S. A. Rice (John Wiley \& Sons, Hoboken, NJ, 2005), Vol. 130A (and references therein).

${ }^{48}$ Kinetics and Nonlinear Dynamics of Complex Many Body Systems, Advances in Chemical Physics, edited by R. S. Berry, T. Komatsuzaki, and D. M. Leitner (John Wiley \& Sons, Hoboken, NJ, in press).

${ }^{49}$ A. Lichtenberg and M. Lieberman, Regular and Chaotic Dynamics (Springer, New York, 1992).

${ }^{50}$ A. Deprit, Celest. Mech. 1, 12 (1969).

${ }^{51}$ T. Bartsch, R. Hernandez, and T. Uzer, Phys. Rev. Lett. 95, 058301 (2005).

${ }^{52}$ T. Bartsch, T. Uzer, and R. Hernandez, J. Chem. Phys. 123, 204102 (2005).

${ }^{53}$ T. Bartsch, T. Uzer, J. M. Moix, and R. Hernandez, J. Chem. Phys. 124, 244310 (2006).

${ }^{54}$ A. Y. T. Leung and Q. C. Zhang, J. Sound Vib. 266, 261 (2003).

${ }^{55}$ S. Kawai, A. D. Bandrauk, C. Jaffé, T. Bartsch, J. Palacián, and T. Uzer, J. Chem. Phys. 126, 164306 (2007).

${ }^{56}$ K. Müller and L. D. Brown, Theor. Chim. Acta 53, 75 (1979).

${ }^{57}$ S. Wiggins, Normally Hyperbolic Invariant Manifolds in Dynamical Systems (Springer-Verlag, New York, 1991).

${ }^{58}$ J. Murdock, Normal Forms and Unfoldings for Local Dynamical Systems, 1st ed. (Springer, New York, 2002).

${ }^{59}$ J. Moser, Commun. Pure Appl. Math. 11, 257 (1958)

${ }^{60}$ S. Kawai and T. Komatsuzaki, J. Chem. Phys. 131, 224506 (2009). 